\title{
ISOMETRIES OF CSL ALGEBRAS
}

\author{
BARUCH SOLEL
}

\begin{abstract}
We show that every Jordan isomorphism of CSL algebras, whose restriction to the diagonal of the algebra is a selfadjoint map, is the sum of an isomorphism and an anti-isomorphism.

It follows that every surjective linear isometry of CSL algebras is the sum of an isomorphism and an anti-isomorphism, followed by a unitary multiplication.
\end{abstract}

\section{INTRODUCTION}

In $[\mathrm{K}], \mathrm{R}$. Kadison proved that every linear isometry of one $C^{*}$-algebra onto another is given by a Jordan $*$-isomorphism followed by a unitary multiplication. (Here a linear map $\varphi$ from a $C^{*}$-algebra $\mathscr{B}_{1}$ into a $C^{*}$-algebra $\mathscr{B}_{2}$ is called a Jordan *-isomorphism if it is one-to-one, surjective, $\varphi\left(x^{*}\right)=\varphi(x)^{*}$ and $\varphi(x y+y x)=\varphi(x) \varphi(y)+\varphi(y) \varphi(x)$ for all $x, y \in \mathscr{B}_{1}$.) Kadison also proved that a Jordan $*$-isomorphism from a von Neumann algebra $\mathscr{B}_{1}$ onto a von Neumann algebra $\mathscr{B}_{2}$ can be decomposed into the sum of a $*$-isomorphism and of a $*$-anti-isomorphism by a central projection.

For nonselfadjoint algebras the following general result was proved in [AS].

Theorem 1.1. Let $\mathscr{U} \subseteq B(H)$ and $\mathscr{B} \subseteq B(K)$ be unital norm closed subalgebras, and let $\varphi: \mathscr{U} \rightarrow \mathscr{B}$ be a surjective linear isometry. Then

(1) $\varphi\left(\mathscr{U} \cap \mathscr{U}^{*}\right)=\mathscr{B} \cap \mathscr{B}^{*}$.

(2) $\varphi\left(x y^{*} z+z y^{*} x\right)=\varphi(x) \varphi(y)^{*} \varphi(z)+\varphi(z) \varphi(y)^{*} \varphi(x)$ for every $x, z$ in $\mathscr{U}$ and $y$ in $\mathscr{U} \cap \mathscr{U}^{*}$.

(3) $U=\varphi(I)$ is a unitary operator in $\mathscr{B} \cap \mathscr{B}^{*}$.

If, moreover, $\varphi(I)=I$, then

(4) $\varphi(x y+y x)=\varphi(x) \varphi(y)+\varphi(y) \varphi(x), x, y \in \mathscr{U}$

(5) $\varphi\left(x^{*}\right)=\varphi\left(x^{*}\right)$ for $x \in \mathscr{U} \cap \mathscr{U}^{*}$.

Note that (2) shows that $\varphi$ preserves the partial triple product $\{x, y, z\}=$ $\frac{1}{2}\left(x y^{*} z+z y^{*} x\right), y \in \mathscr{U} \cap \mathscr{U}^{*}, x, z \in \mathscr{U}$. We shall therefore refer to a surjective, one-to-one linear map that satisfies (1)-(3) as a partial triple *isomorphism.

Also, a surjective one-to-one linear map $\varphi: \mathscr{U} \rightarrow \mathscr{B}$ will be called Jordan partial *-isomorphism if it maps $I$ to $I$ and both it and its inverse satisfy

Received by the editors February 12, 1990 and, in revised form, April 30, 1990.

1980 Mathematics Subject Classification (1985 Revision). Primary 47D25; Secondary 46L10, 46L70.

Key words and phrases. Isometry, Jordan isomorphism, reflexive algebra, CSL, von Neumann algebra, nonselfadjoint algebra. 
properties (4) and (5) above. It follows from Theorem 1.1 that every isometry between unital normed closed operator algebras is given by a Jordan partial *-isomorphism followed by a unitary multiplication (thus extending Kadison's result to nonselfadjoint algebras).

The main result of the present paper (Theorem 2.15) is that, when $\mathscr{U}$ and $\mathscr{B}$ are reflexive operator algebras with commutative subspace lattices (called CSL algebras), then every partial Jordan *-isomorphism can be decomposed into the sum of an isomorphism and an anti-isomorphism by a projection in the center of $\mathscr{U}$.

In particular, every isometry from CSL algebra onto another is such a sum followed by a unitary multiplication. For completely distributive CSL algebras a more concrete result was proved by $\mathrm{R}$. Moore and T. Trent in [MT2].

In order to state their result we shall first set some notation and terminology. A Hilbert space $H$ would be assumed to be separable, an operator on $H$ would be assumed to be bounded and a projection is assumed to be orthogonal. A lattice $\mathscr{L}$ of projections is a strongly closed collection of projections that is closed under the usual lattice operations $\vee$ and $\wedge$ and contains 0 and $I$. In this paper we will deal only with commutative lattices (in which the projections commute pairwise). Such a lattice is called a CSL. A nest is a linearly ordered lattice. If $\mathscr{L}$ is a lattice we write $\operatorname{Alg} \mathscr{L}$ for the collection of operators in $B(H)$ which leave invariant the ranges of all of the projections in $\mathscr{L}$, i.e.

$$
\operatorname{Alg} \mathscr{L}=\{T \in B(H):(I-N) T N=0, N \in \mathscr{L}\} .
$$

$\operatorname{Alg} \mathscr{L}$ is a weakly closed subalgebras of $B(H)$, containing $I$. If $\mathscr{A}$ is a subalgebra of $B(H)$ we write

$\mathscr{L} a t_{\mathscr{A}}=\{N: N$ is a projection, $(I-N) T N=0$ for all $T \in \mathscr{A}\}$. If $\mathscr{L}$ is commutative, then $\mathscr{L}$ at $(\mathrm{Alg} \mathscr{L})=\mathscr{L}$ [A, Theorem 1.6.3]. For a projection $E$ we write $E^{\perp}=I-E$ and for a lattice $\mathscr{L}$ we write $\mathscr{L}^{\perp}=\left\{N^{\perp}: N \in \mathscr{L}\right\}$. Note also that $\operatorname{Alg}\left(\mathscr{L}^{\perp}\right)=(\operatorname{Alg} \mathscr{L})^{*}$ and $(\operatorname{Alg} \mathscr{L})^{*} \cap \operatorname{Alg} \mathscr{L}=\mathscr{L}^{\prime}$.

A CSL $\mathscr{L}$ is said to be completely distributive if it satisfies a certain latticetheoretic condition (see [D, Chapter 23]). An alternative characterization of completely distributive CSL was proved by Laurie and Longstaff [LL]. They showed that $\mathscr{L}$ is completely distributive if and only if the linear span of the rank one operators in $\operatorname{Alg} \mathscr{L}$ is $\sigma$-weakly dense in $\operatorname{Alg} \mathscr{L}$.

The main result of [MT2] is the following.

Theorem 1.2 [MT2, Theorem 2.1]. Let $\mathscr{L}_{1}$ and $\mathscr{L}_{2}$ be completely distributive CSL's on a Hilbert space $H$. Let $\theta: \operatorname{Alg} \mathscr{L}_{1} \rightarrow \operatorname{Alg} \mathscr{L}_{2}$ be a linear surjective isometry. Let $U=\theta(I)$ and write $\varphi(T)=U^{*} \theta(T)$. Then there exist projections $E_{1} \in \mathscr{L}_{1} \cap \mathscr{L}_{1}^{\perp}, E_{2} \in \mathscr{L}_{2} \cap \mathscr{L}_{2}^{\perp}$ and an involution $J$ such that

(1) $\varphi$ restricted to $\left(\operatorname{Alg} \mathscr{L}_{1}\right) E_{1}$, is implemented by a unitary operator $V$ (i.e. $\left.\varphi(T)=V^{*} T V\right)$ such that $N \mapsto V^{*} N V$ is an order isomorphism of $\mathscr{L}_{1} E_{1}$ onto $\mathscr{L}_{2} E_{2}$.

(2) For $\varphi$, restricted to $\left(\operatorname{Alg} \mathscr{L}_{1}\right) E_{1}^{\perp}$, there is a unitary operator $W$ such that $\varphi(T)=W^{*} J T J W$ and the map $N \mapsto W^{*} J N J W$ is an order isomorphism from $\mathscr{L}_{1} E_{1}^{\perp}$ onto $\mathscr{L}_{2}^{\perp} E_{2}^{\perp}$.

The proof of this result in [MT2] uses heavily the fact that there are many rank one operators in $\operatorname{Alg} \mathscr{L}$. In this paper we deal with general CSL lattices and, in the general case, $\operatorname{Alg} \mathscr{L}$ might contain no rank one operators. Therefore 
the methods are completely different. As we remark at the end of the paper, our main result (Theorem 2.15) can be combined with [DP, Theorem 2.1] to yield an alternative proof for Theorem 1.2.

For the special case where $\mathscr{L}_{1}$ and $\mathscr{L}_{2}$ are nests Theorem 1.2 was proved, independently (and using different methods), in [AS and MT1]. In this case either $E_{1}=0$ or $E_{1}=I\left(\right.$ as $\left.\mathscr{L} \cap \mathscr{L}^{\perp}=\{0, I\}\right)$.

\section{JORDAN PARTIAL $*$-ISOMORPHISMS}

We now fix two CSL's $\mathscr{L}$ and $\mathscr{L}_{1}$ and $\varphi: \operatorname{Alg} \mathscr{L} \rightarrow \operatorname{Alg} \mathscr{L}_{1}$, a Jordan partial *-isomorphism; i.e. $\varphi$ is linear, one-to-one, surjective, $\varphi(I)=I, \varphi\left(x^{*}\right)=\varphi(x)^{*}$ for $x \in \operatorname{Alg} \mathscr{L} \cap(\operatorname{Alg} \mathscr{L})^{*}, \varphi(x y+y x)=\varphi(x) \varphi(y)+\varphi(y) \varphi(x)$ for $x, y \in$ $\operatorname{Alg} \mathscr{L}$ and its inverse also satisfies these properties. We have the following:

(I) $\varphi\left(\operatorname{Alg} \mathscr{L} \cap(\operatorname{Alg} \mathscr{L})^{*}\right)=\operatorname{Alg} \mathscr{L}_{1} \cap\left(\operatorname{Alg} \mathscr{L}_{1}\right)^{*}$; i.e. $\varphi\left(\mathscr{L}^{\prime}\right)=\mathscr{L}_{1}^{\prime}$.

(II) As $\varphi$ preserves commutativity, $\varphi\left(\mathscr{L}^{\prime \prime}\right)=\mathscr{L}_{1}^{\prime \prime}$. Thus $\varphi$, restricted to $\mathscr{L}^{\prime \prime}$ (which is an abelian von Neumann algebra), is a $*$-isomorphism. In particular, for every collection of projections $\left\{E_{\alpha}\right\} \subseteq \mathscr{L}^{\prime \prime}, \varphi\left(\bigvee E_{\alpha}\right)=\bigvee \varphi\left(E_{\alpha}\right)$ and $\varphi\left(\bigwedge E_{\alpha}\right)=\bigwedge \varphi\left(E_{\alpha}\right)$.

(III) For all $R, S, T \in \operatorname{Alg} \mathscr{L}$ we have $\varphi(R S T+T S R)=\varphi(R) \varphi(S) \varphi(T)+$ $\varphi(T) \varphi(S) \varphi(R)$ [AS, Corollary 2.11].

We write $\mathscr{A}$ for $\operatorname{Alg} \mathscr{L}$ and for $\mathscr{A}_{1}$ for $\operatorname{Alg} \mathscr{L}_{1}$. For a subset $S \subseteq H$ we write [S] for the closed linear subspace spanned by $S$.

Lemma 2.1. The following are equivalent for a projection $E \in \mathscr{L}^{\prime \prime}$.

(1) $E=E_{1}-E_{2}$ for some $E_{i} \in \mathscr{L}, i=1,2$.

(2) For every $T, S$ in alg $\mathscr{L}, E T E S E=E T S E$.

(3) For every $T, S$ in alg $\mathscr{L}$,

$$
E T E S E+E S E T E=E T S E+E S T E .
$$

Proof. (1) $\Rightarrow(2)$. For every $T, S$ in alg $\mathscr{L}$ and $E=E_{1}-E_{2}, E_{1}, E_{2} \in \mathscr{L}$ we have

$$
E T=E_{1}\left(1-E_{2}\right) T=E_{1}\left(1-E_{2}\right) T\left(1-E_{2}\right)
$$

and $S E=S E_{1}\left(1-E_{2}\right)=E_{1} S E$; hence $E T S E=E T E S E . \quad(2) \Rightarrow$ (3) is obvious.

$(2) \Rightarrow(1)$. Assume (2) holds. Write $E_{1}$ for the orthogonal projection onto [ $\mathscr{A} E(H)]$. Then $E_{1} \in \mathscr{L}$. It is left to show that $E_{1}-E \in \mathscr{L}$. Fix $T \in \mathscr{A}$ and $x$ in $\left(E_{1}-E\right)(H)$. Suppose $x=S E y$ for some $S \in \mathscr{A}$ (and $E X=0$ ). Clearly $T x=T S E y \in E_{1}(H)$ and also $E T x=E T S E y=E T E S E y=E T E x=0$. Hence $T x \in\left(E_{1}-E\right)(H)$. As vectors of the form $S E y$ are dense in $\left(E_{1}-E\right)(H)$ we see that $T x \in\left(E_{1}-E\right)(H)$ for all $x \in\left(E_{1}-E\right)(H)$.

(3) $\Rightarrow(2)$. Let $\left\{E_{i}\right\}_{i=1}^{\infty}$ be a countable subset of $\mathscr{L}$ that is strongly dense in $\mathscr{L}$ and such that $E_{1}=0, E_{2}=I$. As in [A, Proof Theorem 2.2.3] we fix $n \geq 1$ and for each $n$-tuple $\alpha=\left(\alpha_{1}, \ldots, \alpha_{n}\right)$ with $\alpha_{i}=+1$ or -1 we define $E^{\alpha}=E_{1}^{\alpha_{1}} E_{1}^{\alpha_{2}} \cdots E_{n}^{\alpha_{n}}$ where $E_{i}^{1}=E_{i}$ and $E_{i}^{-1}=I-E_{i}$.

As $\alpha$ runs over all $n$-tuples $E^{\alpha}$ runs over the atoms of the Boolean algebra generated by $\left\{E_{1}, \ldots, E_{n}\right\}$. Also, if $\alpha \neq \beta, E^{\alpha} E^{\beta}=0$. We assume here that $E T E S E+E S E T E=E T S E+E S T E$ for all $T, S \in \mathscr{A}$. Apply this to $E^{\alpha} T$ and $S E^{\beta}$ for some $\alpha \neq \beta$ to get $E E^{\alpha} T S E^{\beta} E=E E^{\alpha} T E S E^{\beta} E$ (as $\left.S E^{\beta} E^{\alpha} T=S E^{\beta} E E^{\alpha} T=0\right)$. Write $K=E T S E-E T E S E$. Then $E^{\alpha} K E^{\beta}=0$ 
for all $\alpha \neq \beta$. As $\sum E^{\alpha}=I$ we get

$$
K=\sum_{\alpha} E^{\alpha} K E^{\alpha} \quad \text { and } \quad K \in\left\{E_{1}, \ldots, E_{n}\right\}^{\prime} .
$$

We have, for $1 \leq i \leq n$,

$$
K E_{i}=E T(1-E) S E E_{i}=E T(1-E) E_{i} S E_{i} E
$$

and

$$
\begin{aligned}
K\left(I-E_{i}\right) & =\left(I-E_{i}\right) K\left(I-E_{i}\right)=\left(1-E_{i}\right) E T(I-E) S E\left(I-E_{i}\right) \\
& =E T(I-E)\left(I-E_{i}\right) S\left(I-E_{i}\right) E .
\end{aligned}
$$

Using this repeatedly we get

$$
K E^{\alpha}=E T(1-E) E^{\alpha} S E^{\alpha} E
$$

for all $\alpha$. Hence $K=\sum K E^{\alpha}=E T(1-E)\left(\sum_{\alpha} E^{\alpha} S E^{\alpha}\right) E$. Write $S_{n}=$ $\sum_{\alpha} E^{\alpha} S E^{\alpha}$. Then $S_{n} \in\left\{E_{1}, \ldots, E_{n}\right\}^{\prime}$ and $\left\|S_{n}\right\| \leq\|S\|$. Hence there is a weakly convergent subsequence $S_{n_{k}} \rightarrow S_{0}$. Clearly $S_{0} \in \mathscr{L}^{\prime}$ and thus $K=$ $E T(1-E) S_{0} E$. As $S_{0} \in \mathscr{L}^{\prime}, S_{0} E=E S_{0}$ and $K=0$. This proves (2).

Corollary 2.2. If $E=E_{1}-E_{2}, E_{i} \in \mathscr{L}$, then there are projections $Q_{1}, Q_{2}$, in $\mathscr{L}_{1}$, such that $\varphi(E)=Q_{1}-Q_{2}$.

Proof. With $E$ as above we have

$$
E T E S E+E S E T E=E T S E+E S T E
$$

for every $T, S \in \mathscr{A}$. Since $\varphi$ preserves Jordan products and $\varphi(E R E)=$ $\varphi(E) \varphi(R) \varphi(E)$ for every $R \in \mathscr{A}$, we have

$$
\begin{gathered}
\varphi(E T E S E+E S E T E)=\varphi(E T E) \varphi(E S E)+\varphi(E S E) \varphi(E T E) \\
=\varphi(E) \varphi(T) \varphi(E) \varphi(S) \varphi(E)+\varphi(E) \varphi(S) \varphi(E) \varphi(T) \varphi(E) .
\end{gathered}
$$

But this is equal to

$$
\begin{aligned}
\varphi(E T S E+E S T E) & =\varphi(E) \varphi(T S+S T) \varphi(E) \\
& =\varphi(E) \varphi(S) \varphi(T) \varphi(E)+\varphi(E) \varphi(T) \varphi(S) \varphi(E) .
\end{aligned}
$$

As $\varphi$ is surjective we can use Lemma 2.1 to complete the proof.

A projection $E$ in $\mathscr{L}^{\prime \prime}$ that can be written as $E_{1}-E_{2}$ for some (not necessarily unique) $E_{1}$ and $E_{2}$ in $\mathscr{L}$ is called an interval.

Now fix $N \in \mathscr{L}$. Write $E=\varphi(N)$. By Corollary 2.2, $E=E_{1}-E_{2}$ for some $E_{1}, E_{2} \in \mathscr{L}_{1}$. (Note that the choice of $E_{1}$ and $E_{2}$ is not unique. We choose one possible pair.) Applying Corollary 2.2 to $\varphi^{-1}$ we can find intervals $Q$ and $P$ such that $\varphi(Q)=I-E_{1}$ and $\varphi(P)=E_{2}$. As $E_{2}+\left(I-E_{1}\right)=I-E$, $P+Q=I-N$. Also, as $E_{2}\left(I-E_{1}\right)=0, P Q=0$.

Lemma 2.3. With $N$ fixed in $\mathscr{L}$ and $E, E_{1}, E_{2}, P, Q$ as above we have

$$
\varphi(N T P)=E_{2} \varphi(T) E \quad \text { and } \quad \varphi(N T Q)=E \varphi(T)\left(I-E_{1}\right)
$$

for all $T \in \mathscr{A}$.

Proof. For $T \in \mathscr{A}$ we have $Q T N=0$ (as $N \in \mathscr{L}$ and $Q \leq I-N$ ) and $\left(I-E_{1}\right) \varphi(T) E=\left(I-E_{1}\right) \varphi(T) E_{1} E=0$ (as $E_{1} \in \mathscr{L}_{1}$ ). Using property (III) of $\varphi$ (see the beginning of this section) we now have,

$$
\begin{aligned}
\varphi(N T Q) & =\varphi(N T Q+Q T N)=E \varphi(T)\left(I-E_{1}\right)+\left(I-E_{1}\right) \varphi(T) E \\
& =E \varphi(T)\left(I-E_{1}\right) .
\end{aligned}
$$

The proof for $N T P$ is similar and is omitted. 
Lemma 2.4. Write $F_{1}, F_{2}$ for the projection onto $[N \mathscr{A} P(H)]$ and $[N \mathscr{A} Q(H)]$ respectively. Then $\varphi\left(F_{1}\right)$ and $\varphi\left(F_{2}\right)$ are the projections onto $\left[E \mathscr{A}_{1}{ }^{*} E_{2}(H)\right]$ and $\left[E \mathscr{A}_{1}\left(I-E_{1}\right)(H)\right]$ respectively.

For every $T \in \mathscr{A}$ we have $F_{1} N T P=N T P$. Hence $N T P=F_{1} N T P+$ $N T P F_{1}\left(\right.$ as $\left.F_{1} \leq N \leq I-P\right)$ and, thus, $E_{2} \varphi(T) E=\varphi(N T P)=\varphi\left(F_{1}\right) E_{2} \varphi(T) E$ $+E_{2} \varphi(T) E \varphi\left(F_{1}\right)$. But $\varphi\left(F_{1}\right) E_{2}=\varphi\left(F_{1} P\right)=0$; hence for every $T \in \mathscr{A}$,

$$
E_{2} \varphi(T) E=E_{2} \varphi(T) E \varphi\left(F_{1}\right)
$$

and for every $S \in \mathscr{A}_{1}^{*}$,

$$
E S E_{2}=\varphi\left(F_{1}\right) E S E_{2} .
$$

Hence $\varphi\left(F_{1}\right)$ is larger or equal to the projection onto $\left[E \mathscr{A}_{1}^{*} E_{2}(H)\right]$. If we write $F_{1}^{\prime}$ for the latter projection we have

$$
E T^{*} E_{2}=F_{1}^{\prime} E T^{*} E_{2}
$$

for every $T \in \mathscr{A}_{1}$. We have $E_{2} T E=\left(E T^{*} E_{2}\right)^{*}=\left(F_{1}^{\prime} E T^{*} E_{2}\right)^{*}=E_{2} T E F_{1}^{\prime}=$ $E_{2} T E F_{1}^{\prime}+F_{1}^{\prime} E_{2} T E$ (as $\left.F_{1}^{\prime} \leq E \leq I-E_{2}\right)$. Hence, for $T \in \mathscr{A}_{1}, N \varphi^{-1}(T) P=$ $\varphi^{-1}\left(E_{2} T E\right)=\varphi^{-1}\left(E_{2} T E\right) \varphi^{-1}\left(F_{1}^{\prime}\right)+\varphi^{-1}\left(F_{1}^{\prime}\right) \varphi\left(E_{2} T E\right)=N \varphi^{-1}(T) P \varphi^{-1}\left(F_{1}^{\prime}\right)+$ $\varphi^{-1}\left(F_{1}^{\prime}\right) N \varphi^{-1}(T) P=\varphi^{-1}\left(F_{1}^{\prime}\right) N \varphi^{-1}(T) P$ as $P \varphi^{-1}\left(F_{1}^{\prime}\right)=\varphi^{-1}\left(E_{2} F_{1}^{\prime}\right)=0$. Hence $\varphi^{-1}\left(F_{1}^{\prime}\right) \geq F_{1}$; i.e. $F_{1}^{\prime} \geq \varphi\left(F_{1}\right)$ proving that $\varphi\left(F_{1}\right)$ is equal to $F_{1}^{\prime}$. The proof for $[N \mathscr{A} Q(H)]$ is similar and is omitted.

Lemma 2.5. With the notation of Lemma 2.4, $F_{1} F_{2}=0$.

Proof. For every $T, S$ in $\mathscr{A}$ we have $0=N T P N S Q+N S Q N T P$ as $P N=$ $Q N=0$. Hence

$$
\begin{aligned}
0 & =\varphi(N T P N S Q+N S Q N T P)=\varphi(N T P) \varphi(N S Q)+\varphi(N S Q) \varphi(N T P) \\
& =E_{2} \varphi(T) E E \varphi(S)\left(I-E_{1}\right)+E \varphi(S)\left(I-E_{1}\right) E_{2} \varphi(T) E \\
& =E_{2} \varphi(T) E \varphi(S)\left(I-E_{1}\right)
\end{aligned}
$$

as $\left(I-E_{1}\right) E_{2}=0$. Hence $E_{2} \mathscr{A}_{1} E \mathscr{A}_{1}\left(I-E_{1}\right)=\{0\}$. From Lemma 2.4 it follows that $E_{2} \mathscr{A}_{1} E \varphi\left(F_{2}\right)=\{0\}$; hence $\varphi\left(F_{2}\right) E \mathscr{A}_{1}{ }^{*} E_{2}=\{0\}$. Using Lemma 2.4 again we get $\varphi\left(F_{2}\right) \varphi\left(F_{1}\right)=0$ and, thus, $F_{1} F_{2}=0$.

Note here that both $F_{1}$ and $F_{2}$ are in $\mathscr{L}$ (to see that $F_{1} \in \mathscr{L}$ just observe that for $T, S \in \mathscr{A}, T N S P=N T N S P$; so that $T F_{1}=F_{1} T F_{1}$. The proof for $F_{2}$ is similar). Also $F_{1}+F_{2} \leq N$.

For the projection $N \in \mathscr{L}$ above we associated projections $E, E_{1}, E_{2}, Q$, $P, F_{1}, F_{2}$ etc. This can be done for every projection $L \in \mathscr{L}$ and then we shall write $E(L), E_{1}(L), E_{2}(L)$, etc.

Lemma 2.6. Fix $N \in \mathscr{L}$ as above and let $F_{1}, F_{2}$ be the projections defined above. Then, for every projection $M \in \mathscr{L}$,

(1) $I-\varphi\left(M F_{1}\right) \in \mathscr{L}_{1}$,

(2) $\varphi\left(M F_{2}\right) \in \mathscr{L}_{1}$.

Proof. We shall prove (1). The proof of (2) is similar. Note that from Lemma 2.4 we know:

(i) $E_{2} \mathscr{A}_{1} E\left(I-\varphi\left(F_{1}\right)\right)=\{0\}$,

(ii) $\left(I-\varphi\left(F_{2}\right)\right) E \mathscr{A}_{1}\left(I-E_{1}\right)=\{0\}$, and from Lemma 2.5 ,

(iii) $F_{1} F_{2}=0$. 
Now (ii) and (iii) imply that $\varphi\left(M F_{1}\right) \mathscr{A}_{1}\left(I-E_{1}\right)=\{0\}$. To prove (1) we have to show that

$$
\varphi\left(M F_{1}\right) \mathscr{A}_{1}\left(I-\varphi\left(M F_{1}\right)\right)=\{0\} .
$$

Write $G=M F_{1}$. Then $G \in \mathscr{L}$ and $E(G), E_{1}(G)$, etc. are well defined. Applying (ii)-(iii) to $G$, in place of $N$, we get

(i') $E_{2}(G) \mathscr{A}_{1} E(G)\left(I-\varphi\left(F_{1}(G)\right)\right)=\{0\}$,

(ii') $\left(I-\varphi\left(F_{2}(G)\right)\right) E(G) \mathscr{A}_{1}\left(I-E_{1}(G)\right)=\{0\}$,

(iii') $F_{1}(G) F_{2}(G)=0$.

As $E(G)=\varphi(G) \leq \varphi(N)=E \leq I-E_{2}$ and $E(G)=E_{1}(G)-E_{2}(G)$, it is easy to check that $E(G)=E_{2} \vee E_{1}(G)-E_{2} \vee E_{2}(G)$; hence, replacing $E_{i}(G)$, $i=1,2$, by $E_{i}(G) \vee E_{2}$, we can assume that $E_{i}(G) \geq E_{2}$. (Recall that the choice of $E_{1}(G)$ and $E_{2}(G)$ was arbitrary.)

We now get, from $\left(\mathrm{i}^{\prime}\right), E_{2} \mathscr{A}_{1} E(G)\left(I-\varphi\left(F_{1}(G)\right)\right)=\{0\}$, i.e.

$$
\left(1-\varphi\left(F_{1}(G)\right)\right) E(G) \mathscr{A}_{1}^{*} E_{2}=\{0\} .
$$

But $E(G)=\varphi(G)=\varphi\left(F_{1}\right) \varphi(M)$. Hence $\left(1-\varphi\left(F_{1}(G)\right)\right) \varphi(M) \varphi\left(F_{1}\right) \mathscr{A}_{1}^{*} E_{2}=$ $\{0\}$. As $\varphi\left(F_{1}\right)$ is the projection onto $\left[E \mathscr{A}_{1}{ }^{*} E_{2}(H)\right]$ and $E \geq \varphi\left(F_{1}\right)$, it is the projection onto $\left[\varphi\left(F_{1}\right) \mathscr{A}_{1}{ }^{*} E_{2}(H)\right]$. We therefore have

$$
0=\left(I-\varphi\left(F_{1}(G)\right)\right) \varphi(M) \varphi\left(F_{1}\right)=\left(I-\varphi\left(F_{1}(G)\right)\right) \varphi(G) .
$$

It follows that $G\left(I-F_{1}(G)\right)=0$. But $F_{1}(G) \leq G$; hence $G=F_{1}(G)$. As $F_{2}(G) \leq G-F_{1}(G), F_{2}(G)=0$ and, using (ii') we get

$$
E(G) \mathscr{A}_{1}\left(I-E_{1}(G)\right)=\{0\} .
$$

Also $E(G) \mathscr{A}_{1} E_{2}(G)=\{0\}$ as $E_{2}(G) \in \mathscr{L}_{1}$ and $E_{2}(G) E(G)=0$. Combining this with $(*)$ we have

Hence $\varphi\left(M F_{1}\right)=\varphi(G)=E(G) \in \mathscr{L}_{1}^{\perp}$.

$$
E(G) \mathscr{A}_{1}(I-E(G))=\{0\} .
$$

We shall now write $P_{0}=\bigvee\left\{P \in \mathscr{L} \cap \mathscr{L}^{\perp} ; \mathscr{A} P \subseteq \mathscr{L}^{\prime}\right\}$ and note that $\mathscr{A} P_{0} \subseteq \mathscr{L}^{\prime}$.

Lemma 2.7. For $N$ and $M$ in $\mathscr{L}, F_{1}(N) F_{2}(M) \leq P_{0}$.

Proof. Write $L=F_{1}(N) F_{2}(M) \in \mathscr{L}$. As $L \leq F_{1}(N)$ and $L \leq F_{2}(M)$, Lemma 2.6 implies that $\varphi(L) \in \mathscr{L}_{1} \cap \mathscr{L}_{1}^{\perp}$. Note that $\mathscr{L} \cap \mathscr{L}^{\perp}$ is the set of all projections in $\mathscr{A}^{\prime}$. Thus $\varphi\left(\mathscr{L} \cap \mathscr{L}^{\perp}\right)=\mathscr{L}_{1} \cap \mathscr{L}_{1}^{\perp}$ and thus $L \in \mathscr{L} \cap \mathscr{L}^{\perp}$. In fact, for every $N_{1} \in \mathscr{L} \quad N_{1} L \leq L$ and the same argument shows that $N_{1} L \in \mathscr{L} \cap \mathscr{L}^{\perp}$. Hence $\mathscr{L} L \subseteq \mathscr{L} \cap \mathscr{L}^{\perp}$ from which it easily follows that $\mathscr{A} L \subseteq \mathscr{L}^{\prime}$. Hence $L \leq P_{0}$.

We now write $F_{+}=\bigvee\left\{F_{2}(M): M \in \mathscr{L}\right\}$ and $F_{-}=\bigvee\left\{F_{1}(M): M \in \mathscr{L}\right\}$. If $N \in \mathscr{L}$ and $N \leq F_{+}$then $N=\bigvee\left\{N F_{2}(M): M \in \mathscr{L}\right\}$ and $\varphi(N)=$ $\bigvee\left\{\varphi\left(N F_{2}(M)\right): M \in \mathscr{L}\right\} \in \mathscr{L}_{1}$ (by Lemma 2.6). Similarly whenever $N \in \mathscr{L}$ and $N \leq F_{-}, \varphi(N) \in \mathscr{L}_{1}^{\perp}$. Note also that $F_{+} F_{-} \leq P_{0}$ (by Lemma 2.7). Suppose that $P_{0}=0$ so that $F_{+} F_{-}=0$.

Write $A=F_{+}+F_{-} \in \mathscr{L}$ and note that $\varphi(I-A)=\left(I-\varphi\left(F_{+}\right)\right)-\varphi\left(F_{-}\right)$and $I-\varphi\left(F_{+}\right), \varphi\left(F_{-}\right) \in \mathscr{L}_{1}^{\perp}$.

We can thus apply Lemma 2.5 and Lemma 2.6 with $\mathscr{A}^{*}, \mathscr{A}_{1}^{*}$ replacing $\mathscr{A}$ and $\mathscr{A}_{1}, \mathscr{L}^{\perp}$ and $\mathscr{L}_{1}^{\perp}$ replacing $\mathscr{L}$ and $\mathscr{L}_{1}, \varphi^{*}$ (defined by $\varphi^{*}\left(T^{*}\right)=$ $\left.\varphi(T)^{*}\right)$ replacing $\varphi$ and $I-A, F_{+}$and $F_{-}$replacing $N, Q$ and $P$ respectively to get the following. 
Lemma 2.8. Suppose $P_{0}=0$ and let $A_{+}$and $A_{-}$be the projections onto $\left[(I-A) \mathscr{A}^{*} F_{+}(H)\right]$ and $\left[(I-A) \mathscr{A}^{*} F_{-}(H)\right]$ respectively. Then

(1) $A_{+} A_{-}=0$.

(2) For every projection $M \in \mathscr{L}, \varphi\left((I-M) A_{-}\right) \in \mathscr{L}_{1}$.

Lemma 2.9. $(I-A) \mathscr{A} \subseteq \mathscr{L}^{\prime}$.

Proof. Fix $N \in \mathscr{L}$. Recall that $F_{1}(N)$ is the projection onto $[N A P(H)]$, $F_{2}(N)$ is the projection onto $[N \mathscr{A} Q(H)], Q+P=I-N$ and $F_{1}(N)+F_{2}(N) \leq$ $A$. Hence

$$
(I-A) N \mathscr{A}(I-N)=\{0\} \text {. }
$$

also $(I-N) A N=\{0\}$ (as $N \in \mathscr{L})$. We therefore have

$$
(I-N)(I-A) \mathscr{A} N=\{0\}=N(I-A) \mathscr{A}(I-N) \text {. }
$$

Hence $(I-A) \mathscr{A} \subseteq \mathscr{L}^{\prime}$.

Finally, write $E_{+}=F_{+}+A_{+}$and $E_{-}=F_{-}+A_{-}$.

Lemma 2.10. Suppose $P_{0}=0$. Then

(1) $E_{+}$and $E_{-}$are in $\mathscr{L} \cap \mathscr{L}^{\perp}$.

(2) $E_{+}+E_{-}=I$.

(3) For every $M \in \mathscr{L}, \varphi\left(M E_{+}\right) \in \mathscr{L}_{1}$ and $\varphi\left(M E_{-}\right) \in \mathscr{L}_{1}^{\perp}$.

Proof. First we show that $E_{+} \in \mathscr{L}$. As $F_{+} \in \mathscr{L}$ it suffices to show that $\left(I-E_{+}\right) \mathscr{A} A_{+}=0$. As $I-E_{+}=F_{-}+\left(I-A_{+}\right)(I-A)$, we shall show

(i) $F_{-} \mathscr{A} A_{+}=\{0\}$; and

(ii) $\left(I-A_{+}\right)(I-A) \mathscr{A} A_{+}=\{0\}$.

For (i) note that $A_{+} A_{-}=0$ and $A_{-}$is the projection on $\left[(I-A) \mathscr{A}^{*} F_{-}(H)\right]$; hence $A_{+}(I-A) \mathscr{A}^{*} F_{-}=\{0\}$ and, thus, $F_{-} \mathscr{A}(I-A) A_{+}=0$. As $A_{+}=$ $(I-A) A_{+}$, this proves (i). (ii) follows immediately from the fact that $(I-A) \mathscr{A} \subseteq \mathscr{L}^{\prime}$ (Lemma 2.9). Hence $E_{+} \in \mathscr{L}$. The proof that $E_{-} \in \mathscr{L}$ is almost identical and is omitted.

Now write $A_{0}=I-E_{+}-E_{-} \leq I-F_{+}-F_{-}=I-A$. As $(I-A) \mathscr{A} \subseteq \mathscr{L}^{\prime}$ (Lemma 2.9), also $A_{0} \mathscr{A} \subseteq \mathscr{L}^{\prime}$. Note also that, as $A_{0} A_{+}=A_{0} A_{-}=0$ we have, $A_{0} \mathscr{A}^{*} F_{+}=A_{0} \mathscr{A}^{*} F_{-}=\{0\}$; hence $A_{0} \mathscr{A}^{*} A=\{0\}$ and, thus, $A \mathscr{A} A_{0}=\{0\}$. As $(I-A) \mathscr{A} \subseteq \mathscr{L}^{\prime}$, we have $(I-A) \mathscr{A} A_{0} \subseteq \mathscr{L}^{\prime}$. Therefore $\mathscr{A} A_{0}=A \mathscr{A} A_{0}+$ $(I-A) \mathscr{A} A_{0} \subseteq \mathscr{L}^{\prime}$. Hence we have $\mathscr{A} A_{0} \subseteq \mathscr{L}^{\prime}$ and $A_{0} \mathscr{A} \subseteq \mathscr{L}^{\prime}$ and this implies that $A_{0} \in \mathscr{A}^{\prime}$ (as $\left(I-A_{0}\right) \mathscr{A} A_{0} \subseteq\left(I-A_{0}\right) \mathscr{L}^{\prime} A_{0}=\{0\}$ and, similarly $\left.A_{0} \mathscr{A}\left(I-A_{0}\right)=\{0\}\right)$; i.e. $A_{0} \leq P_{0}=0$.

This shows that $E_{+}+E_{-}=I$ and, as $E_{+}, E_{-} \in \mathscr{L}$ we have $E_{+}, E_{-} \in$ $\mathscr{L} \cap \mathscr{L}^{\perp}$. This proves parts (1) and (2). For (3) we fix $M \in \mathscr{L}$. We want to show that $\varphi\left(M E_{+}\right) \in \mathscr{L}_{1}$. As $E_{+} \in \mathscr{L} \cap \mathscr{L}^{\perp} \subseteq \mathscr{A}^{\prime}$ we can replace $\mathscr{L}$ by $\mathscr{L} E^{+}$and $\mathscr{A}$ by $\mathscr{A} E_{+}$; i.e. we assume $E_{+}=I$. Then $F_{+}+A_{+}=I$.

Note first that by Lemma $2.8(2)$ we know that $\varphi\left((I-M) A_{+}\right) \in \mathscr{L}_{1}^{\perp}$. Hence we have

(*) $\varphi\left((I-M) A_{+}\right) \mathscr{A}_{1} \varphi\left(M A_{+}\right)=\varphi\left((I-M) A_{+}\right) \mathscr{A}_{1} \varphi\left((I-M) A_{+}\right) \varphi\left(M A_{+}\right)=\{0\}$.

For every $T \in \mathscr{A}, F_{+}(I-M) T M A_{+}=0$ (as $M \in \mathscr{L}$ ) and $M A_{+} T(I-M) F_{+}=$ 0 (as $F_{+} \in \mathscr{L}$ and $A_{+} F_{+}=0$ ). Hence

$$
\begin{aligned}
0 & =\varphi\left(F_{+}(I-M) T M A_{+}+M A_{+} T(I-M) F_{+}\right) \\
& =\varphi\left(F_{+}(I-M)\right) \varphi(T)\left(M A_{+}\right)+\varphi\left(M A_{+}\right) \varphi(T) \varphi\left((I-M) F_{+}\right) .
\end{aligned}
$$


As $\varphi\left(F_{+}\right) \in \mathscr{L}$ and $\varphi\left(A_{+}\right) \varphi\left(F_{+}\right)=0, \varphi\left(M A_{+}\right) \varphi(T) \varphi\left((I-M) F_{+}\right)=0$. Hence $\varphi\left(F_{+}(I-M)\right) \mathscr{A}_{1} \varphi\left(M A_{+}\right)=\{0\}$.

As $I-M=\left(A_{+}+F_{+}\right)(I-M)=A_{+}(I-M)+F_{+}(I-M)$, combining this with $(*)$ we get

$$
\varphi(I-M) \mathscr{A}_{1} \varphi\left(M A_{+}\right)=\{0\} .
$$

As $\varphi\left(M F_{+}\right) \in \mathscr{L}_{1}$ (see the discussion following Lemma 2.7),

$$
\varphi(I-M) \mathscr{A}_{1} \varphi(M)=\varphi(I-M) \mathscr{A}_{1}\left(\varphi\left(M A_{+}\right)+\varphi\left(M F_{+}\right)\right)=\{0\} .
$$

Thus $\varphi\left(M A_{+}\right) \in \mathscr{L}_{1}$.

The proof that $\varphi\left(M A_{-}\right) \in \mathscr{L}^{\perp}$ is similar and is omitted.

Proposition 2.11. Let $\varphi: \mathscr{A} \rightarrow \mathscr{A}_{1}$ be a Jordan partial *-isomorphism of $\mathscr{A}$ onto $\mathscr{A}_{1}$. Then there are projections $E_{+}, E_{-}$and $P_{0}$ in $\mathscr{L} \cap \mathscr{L}^{\perp}$ such that $E_{+}+E_{-}+P_{0}=I$ and

(1) $A P_{0}=\mathscr{L}^{\prime} P_{0}$ (hence $\left.\varphi\left(\mathscr{L} P_{0}\right) \subseteq \mathscr{L}_{1} \cap \mathscr{L}_{1}^{\perp}\right)$;

(2) for every $M \in \mathscr{L}, \varphi\left(M E_{+}\right) \in \mathscr{L}_{1}$ and, in fact, $\varphi\left(\mathscr{L} E_{+}\right)=\mathscr{L}_{1} \varphi\left(E_{+}\right)$; and

(3) for every $M \in \mathscr{L}, \varphi\left(M E_{-}\right) \in \mathscr{L}_{1}^{\perp}$ and, in fact $\varphi\left(\mathscr{L} E_{-}\right)=\mathscr{L}_{1}^{\perp} \varphi\left(E_{-}\right)$.

Proof. Let $P_{0}=\bigvee\left\{P \in \mathscr{L} \cap \mathscr{L}^{\perp}: \mathscr{A} P \subseteq \mathscr{L}^{\prime} P\right\}$ (as above) and replace $\mathscr{A}, \mathscr{A}_{1}$, $\mathscr{L}, \mathscr{L}_{1}$ by $\mathscr{A} P_{0}^{\perp}, \mathscr{A}_{1} \varphi\left(P_{0}^{\perp}\right), \mathscr{L} P_{0}^{\perp}, \mathscr{L}_{1} \varphi\left(P_{0}^{\perp}\right)$ respectively; i.e. assume $P_{0}=$ 0 . Then let $E_{+}$and $E_{-}$be the projections constructed above. By Lemma 2.10 we have $E_{+}+E_{-}=I$. Part (1) now follows from the definition of $P_{0}$. From Lemma 2.10 we know that $\varphi\left(\mathscr{L} E_{+}\right) \subseteq \mathscr{L}_{1} \varphi\left(E_{+}\right)$and $\varphi\left(\mathscr{L} E_{-}\right) \subseteq \mathscr{L}_{1}^{\perp} \varphi\left(E_{-}\right)$. Now write $P_{0}^{\prime}=\bigvee\left\{P \in \mathscr{L}_{1}^{\prime} \cap \mathscr{L}_{1}^{\perp}: \mathscr{A}_{1} P \subseteq \mathscr{L}_{1}^{\prime} P\right\}$. It is clear that $\varphi\left(P_{0}\right)=P_{0}^{\prime}$ and since we assume that $P_{0}=0$, also $P_{0}^{\prime}=0$. If $E_{+}^{\prime}$ and $E_{-}^{\prime}$ are the projections constructed for $\varphi^{-1}$ then $E_{+}^{\prime}+E_{-}^{\prime}=I$. If $\varphi\left(\mathscr{L} E_{+}\right) \neq \mathscr{L}_{1} \varphi\left(E_{+}\right)$ then there exists an element $G_{0} \in \mathscr{L}_{1}$ such that $G_{0} \leq \varphi\left(E_{+}\right)$and $G_{0} \notin$ $\varphi\left(\mathscr{L} E_{+}\right)$. By Lemma $2.10, \varphi^{-1}\left(G_{0} E_{+}^{\prime}\right) \in \mathscr{L}$ and $\varphi^{-1}\left(G_{0} E_{-}^{\prime}\right) \in \mathscr{L}^{\perp}$. Now, if $G_{0} E_{-}^{\prime}=0$, then $\varphi^{-1}\left(G_{0}\right)=\varphi^{-1}\left(G_{0} E_{+}^{\prime}\right) \in \mathscr{L}$ and also $\varphi^{-1}\left(G_{0}\right) \leq E_{+}$; hence $\varphi^{-1}\left(G_{0}\right) \in \mathscr{L} E_{+}$, and we arrive at a contradiction. Therefore $G_{0} E_{-}^{\prime} \neq$ 0 and we write $G=G_{0} E_{-}^{\prime}$. Then $G \leq \varphi\left(E_{+}\right)$and $\varphi^{-1}(G) \in \mathscr{L}^{\perp}$. Thus $I-\varphi^{-1}(G) \in \mathscr{L}$ and, by Lemma 2.10, $\varphi\left(E_{+}\right)-G=\varphi\left(\left(I-\varphi^{-1}(G)\right) E_{+}\right) \in \mathscr{L}_{1}$. Since $E_{+} \in \mathscr{L} \cap \mathscr{L}^{\perp}$ (Lemma 2.10), $\varphi\left(E_{+}\right) \in \mathscr{L}_{1} \cap \mathscr{L}_{1}^{\perp}$. Combining these facts we get $G=\varphi\left(E_{+}\right)\left(I-\left(\varphi\left(E_{+}\right)-G\right)\right) \in \mathscr{L}_{1}^{\perp}$; hence $G \in \mathscr{L}_{1} \cap \mathscr{L}_{1}^{\perp}$. Thus $\varphi^{-1}(G) \in \mathscr{L} \cap \mathscr{L}^{\perp}$ and, since $G_{0}=G_{0} E_{+}^{\prime}+G_{0} E_{-}^{\prime}=G_{0} E_{+}^{\prime}+G$, we have $\varphi^{-1}\left(G_{0}\right)=\varphi^{-1}\left(G_{0} E_{+}^{\prime}\right)+\varphi^{-1}(G) \in \mathscr{L}$. Since $\varphi^{-1}\left(G_{0}\right) \leq E_{+}, \varphi^{-1}\left(G_{0}\right) \in \mathscr{L} E_{+}$. This contradicts the choice of $G_{0}$ and proves that $\varphi\left(\mathscr{L} E_{+}\right)=\mathscr{L}_{1} \varphi\left(E_{+}\right)$. The fact that $\varphi\left(\mathscr{L} E_{-}\right)=\mathscr{L}_{1}^{\perp} \varphi\left(E_{-}\right)$is proved in a similar way.

Let $E_{+}, E_{-}$and $P_{0}$ be as in Proposition 2.11. We first assume that $E_{+}=I$ (i.e. $\left.E_{-}=P_{0}=0\right)$. Then $\varphi(\mathscr{L})=\mathscr{L}_{1}$. The following argument was used in [MT2].

Since $\mathscr{L}^{\prime \prime}$ is a commutative von Neumann algebra there is a maximal abelian selfadjoint algebra (i.e. a masa) $\mathscr{R}$ such that $\mathscr{L}^{\prime \prime} \subseteq \mathscr{R} \subseteq \mathscr{L}^{\prime}=\mathscr{A} \cap \mathscr{A}^{*}$. The restriction of $\varphi$ to $\mathscr{R}$ is a Jordan $*$-isomorphism and therefore its image, $\varphi(\mathscr{R})$ is a abelian selfadjoint algebra, written $\mathscr{R}_{1}$. As $\varphi^{-1}$, restricted to $\mathscr{L}_{1}^{\prime}$, is Jordan *-isomorphism of the von Neumann algebra $\mathscr{L}_{1}^{\prime}$ onto $\mathscr{L}^{\prime}$ it preserves commutativity. Thus if $T \in \mathscr{R}_{1}^{\prime} \subseteq \mathscr{L}_{1}^{\prime}$ then $\varphi^{-1}(T) \in \mathscr{R}^{\prime}=\mathscr{R}$; hence 
$T \in \mathscr{R}_{1}$. This shows that $\mathscr{R}_{1}=\varphi(\mathscr{R})$ is a masa. Now the restriction of $\varphi$ to $\mathscr{R}$ is a Jordan $*$-isomorphism of masa's and, thus, is a $*$-isomorphism. By [KR, Theorem 9.3.1] we know that $\varphi$, restricted to $\mathscr{R}$, can be implemented by some unitary operator $V$; i.e. $\varphi(T)=V^{*} T V, T \in \mathscr{R}$.

Since $\varphi(\mathscr{L})=\mathscr{L}_{1}, V^{*} \mathscr{L} V=\mathscr{L}_{1}$ and $V^{*} \mathscr{A} V=\mathscr{A}_{1}$. If we now write $\psi(T)=V \varphi(T) V^{*}$ then $\psi$ is a Jordan partial $*$-isomorphism from $\mathscr{A}$ onto itself leaving $\mathscr{R}$ (and, in particular, $\mathscr{L}^{\prime \prime}$ ) elementwise fixed.

Lemma 2.12. Suppose $E_{+}=I$ and $\psi$ is as above. Then for every interval $E_{2}-E_{1}$ (with $E_{1}, E_{2} \in \mathscr{L}$ and $E_{1} \leq E_{2}$ ) and $T \in \operatorname{Alg} \mathscr{L}$ we have

$$
\psi\left(T\left(E_{2}-E_{1}\right)\right)=\psi(T)\left(E_{2}-E_{1}\right) \quad \text { and } \quad \psi\left(\left(E_{2}-E_{1}\right) T\right)=\left(E_{2}-E_{1}\right) \psi(T) .
$$

Proof. We have $T E_{2}\left(I-E_{1}\right)=\left(E_{2}-E_{1}\right) T\left(E_{2}-E_{1}\right)+E_{1} T\left(E_{2}-E_{1}\right)$ (as $\left.\left(I-E_{2}\right) T\left(E_{2}-E_{1}\right)=0\right)$. Also $\left(E_{2}-E_{1}\right) T E_{1}=0\left(\right.$ as $\left.E_{1} \in \mathscr{L}\right)$ and thus

$$
\begin{aligned}
\psi( & \left.T\left(E_{2}-E_{1}\right)\right) \\
& =\psi\left(\left(E_{2}-E_{1}\right) T\left(E_{2}-E_{1}\right)\right)+\psi\left(E_{1} T\left(E_{2}-E_{1}\right)+\left(E_{2}-E_{1}\right) T E_{1}\right) \\
& =\left(E_{2}-E_{1}\right) \psi(T)\left(E_{2}-E_{1}\right)+E_{1} \psi(T)\left(E_{2}-E_{1}\right)+\left(E_{2}-E_{1}\right) \psi(T) E_{1} \\
& =\left(E_{2}-E_{1}\right) \psi(T)\left(E_{2}-E_{1}\right)+E_{1} \psi(T)\left(E_{2}-E_{1}\right) \\
& =\psi(T)\left(E_{2}-E_{1}\right) .
\end{aligned}
$$

The other statement is proved in a similar way.

We shall also need the following lemma.

Lemma 2.13. For every $N \in \mathscr{L}$ we write $G_{1}(N)$ for the projection onto $[N \mathscr{A}(I-N)(H)]$ and $G_{2}(N)$ for the projection onto $\left[(I-N) \mathscr{A}^{*} N(H)\right]$. Let $G_{1}=\bigvee\left\{G_{1}(N): N \in \mathscr{L}\right\}$ and $G_{2}=\bigvee\left\{G_{2}(N): N \in \mathscr{L}\right\}$. Then $G_{1} \vee G_{2} \geq$ $I-P_{0}$. Hence if $P_{0}=0$ then $G_{1} \vee G_{2}=I$.

Proof. Write $F=I-G_{1} \vee G_{2}$. As $F G_{1}=F G_{2}=0$ we have $F N \mathscr{A}(I-N)=$ 0 and $F(I-N) \mathscr{A}^{*} N=0$ for every $N \in \mathscr{L}$. Hence $N(F \mathscr{A})(I-N)=$ $N(\mathscr{A} F)(I-N)=0$ for every $N \in \mathscr{L}$. It follows that both $F \mathscr{A}$ and $\mathscr{A} F$ are contained in $\mathscr{A}^{*}$. As both are also contained in $\mathscr{A}$, we have $F \mathscr{A} \subseteq \mathscr{L}^{\prime}$ and $\mathscr{A} F \subseteq \mathscr{L}^{\prime}$ which implies that $F \leq P_{0}$.

Proposition 2.14. Assume that $E_{+}=I$ and $\psi$ is as above then $\psi$ is multiplicative.

Proof. From Lemma 2.13 it follows that we can find sequences $\left\{N_{i}\right\}_{i=1}^{\infty}$ and $\left\{M_{i}\right\}_{i=1}^{\infty}$ of projections in $\mathscr{L}$ such that $\left(\bigvee_{i} G_{1}\left(N_{i}\right)\right) \vee\left(\bigvee_{i} G_{2}\left(M_{i}\right)\right)=I$. We can, therefore, find a sequence of projections $\left\{E_{i}\right\}$ in $\mathscr{L}^{\prime \prime}$ such that:

(i) each $E_{i}$ is an interval in $\mathscr{L}$. (Note that $G_{1}(N) \in \mathscr{L}$ and $G_{2}(N) \in \mathscr{L}^{\perp}$ for every $N \in \mathscr{L}$ ).

(ii) For $i \neq j, E_{i} E_{j}=0$;

(iii) $\sum_{i} E_{i}=I$; and

(iv) For each $i$ there is some $N \in \mathscr{L}$ such that either $E_{i} \leq G_{1}(N)$ or $E_{i} \leq G_{2}(N)$.

Now fix $T, S \in \mathscr{A}$. For $i \neq j$ we have

$$
\begin{aligned}
\psi\left(E_{i} T S E_{j}\right) & =\psi\left(E_{i} T S E_{j}+S E_{j} E_{i} T\right)=\psi\left(E_{i} T\right) \psi\left(S E_{j}\right)+\psi\left(S E_{j}\right) \psi\left(E_{i} T\right) \\
& =E_{i} \psi(T) \psi(S) E_{j}+\psi(S) E_{j} E_{i} \psi(T)=E_{i} \psi(T) \psi(S) E_{j}
\end{aligned}
$$


(We use Lemma 2.12 to see that $\psi\left(E_{i} T\right)=E_{i} \psi(T)$ and $\psi\left(S E_{j}\right)=\psi(S) E_{j}$.) Hence

$$
\psi\left(E_{i} T S E_{j}\right)=E_{i} \psi(T) \psi(S) E_{j}, \quad i \neq j .
$$

Suppose $E_{i}$ is such that $E_{i} \leq G_{1}(N)$ for some $N \in \mathscr{L}$. For every $R \in \mathscr{A}$ we have

$\psi\left(E_{i} T E_{i} S E_{i} N R(I-N)\right)=\psi\left(E_{i} T E_{i} S E_{i} N R(I-N)\right)+\psi\left(N R(I-N) E_{i} S E_{i} T E_{i}\right)$

(as $\left.E_{i} \leq G_{1}(N) \leq N\right)$. Since $\psi$ is a Jordan isomorphism,

$$
\begin{aligned}
& \psi\left(E_{i} T E_{i} S E_{i} N R(I-N)\right)=\psi\left(E_{i} T E_{i}\right) \psi\left(E_{i} S E_{i}\right) \psi(N R(I-N)) \\
& \quad+\psi(N R(I-N)) \psi\left(E_{i} S E_{i}\right) \psi\left(E_{i} T E_{i}\right)=E_{i} \psi(T) E_{i} \psi(S) E_{i} N \psi(R)(I-N) \\
& \quad+N \psi(R)(I-N) E_{i} \psi(S) E_{i} \psi(T) E_{i}=E_{i} \psi(T) E_{i} \psi(S) E_{i} N \psi(R)(I-N) .
\end{aligned}
$$

We get

$$
\psi\left(E_{i} T E_{i} S E_{i} N R(I-N)\right)=E_{i} \psi(T) E_{i} \psi(S) E_{i} N \psi(R)(I-N)
$$

and, replacing $S$ by $T E_{i} S$ and $T$ by $I$ we get

$$
\begin{aligned}
\psi\left(E_{i} T E_{i} S E_{i} N R(I-N)\right) & =E_{i} \psi\left(T E_{i} S\right) E_{i} N \psi(R)(I-N) \\
& =\psi\left(E_{i} T E_{i} S E_{i}\right) N \psi(R)(I-N)
\end{aligned}
$$

and from these two equations we conclude:

$$
E_{i} \psi(T) E_{i} \psi(S) E_{i} N \psi(R)(I-N)=\psi\left(E_{i} T E_{i} S E_{i}\right) N \psi(R)(I-N) .
$$

Since this holds for all $R \in \mathscr{A}$ and since $E_{i}=E_{i} G_{1}(N)$ we get (recall that $G_{1}(N)$ is the projection onto $\left.[N \mathscr{A}(I-N)(H)]\right)$,

$$
\psi\left(E_{i} T E_{i} S E_{i}\right)=E_{i} \psi(T) E_{i} \psi(S) E_{i} .
$$

Using the fact that $\psi$ is a Jordan homomorphism again we get

$$
\begin{aligned}
& \psi\left(E_{i} T S E_{i}+E_{i} S E_{i} T E_{i}\right)=E_{i} \psi\left(E_{i} T S E_{i}+S E_{i} E_{i} T\right) E_{i} \\
& \quad=E_{i}\left(\psi\left(E_{i} T\right) \psi\left(S E_{i}\right)+\psi\left(S E_{i}\right) \psi\left(E_{i} T\right)\right) E_{i} \\
& \quad=E_{i} \psi(T) \psi(S) E_{i}+E_{i} \psi(S) E_{i} \psi(T) E_{i}
\end{aligned}
$$

and, using (**) with $T$ and $S$ reserved, we have

$$
\psi\left(E_{i} T S E_{i}+E_{i} S E_{i} T E_{i}\right)=E_{i} \psi(T) \psi(S) E_{i}+\psi\left(E_{i} S E_{i} T E_{i}\right) .
$$

Hence

$$
\psi\left(E_{i} T S E_{i}\right)=E_{i} \psi(T) \psi(S) E_{i} .
$$

This was shown to hold for $E_{i}$ with the property that $E_{i} \leq G_{1}(N)$. We wish to show it also for $E_{i}$ satisfying $E_{i} \leq G_{2}(N)$ for some $N \in \mathscr{L}$. For that simply replace $\mathscr{A}$ by $\mathscr{A}^{*}, \psi$ by $\psi^{*}\left(\right.$ where $\left.\psi^{*}(T)=\psi(T)^{*}, T \in \mathscr{A}^{*}\right), T$ by $S^{*}$ and $S$ by $T^{*}$ and note that $G_{2}(N)$ is now $G_{1}(I-N)$ (with $\mathscr{A}^{*}$ in place of $\mathscr{A})$. We now get,

$$
\psi^{*}\left(E_{i} S^{*} T^{*} E_{i}\right)=E_{i} \psi^{*}\left(S^{*}\right) \psi^{*}\left(T^{*}\right) E_{i}
$$

and, thus, by taking adjoints, $\psi\left(E_{i} T S E_{i}\right)=E_{i} \psi(T) \psi(S) E_{i}$; so that $(* * *)$ holds for every $i$. 
Now we write

$$
\psi(T S)=\sum_{i j} E_{i} \psi(T S) E_{j}=\sum_{i} E_{i} \psi(T S) E_{i}+\sum_{i \neq j} E_{i} \psi(T S) E_{j}
$$

and, using $(*),(* * *)$, we conclude

$$
\psi(T S)=\sum_{i} E_{i} \psi(T) \psi(S) E_{i}+\sum_{i \neq j} E_{i} \psi(T) \psi(S) E_{j}=\psi(T) \psi(S) .
$$

We are now ready to prove the main theorem.

Theorem 2.15. Let $\mathscr{L}$ and $\mathscr{L}_{1}$ be commutative lattices of projections acting on a separable Hilbert space $H$. Let $\theta: \operatorname{Alg} \mathscr{L} \rightarrow \operatorname{Alg} \mathscr{L}_{1}$ be a partial triple product isomorphism onto $\mathrm{Alg} \mathscr{L}_{1}$. (In particular this holds for a linear surjective isometry.) Let $U=\theta(I)$ and $\varphi: \operatorname{Alg} \mathscr{L} \rightarrow \operatorname{Alg} \mathscr{L}_{1}$ be defined by $\varphi(T)=$ $U^{*} \theta(T), T \in \operatorname{Alg} \mathscr{L}_{1}$. Then there is a projection $E \in \mathscr{L} \cap \mathscr{L}^{\perp} \subseteq(\mathrm{Alg} \mathscr{L})^{\prime}$ such that :

(1) $\varphi$, restricted to $(\mathrm{Alg} \mathscr{L}) E$, is an isomorphism of $(\mathrm{Alg} \mathscr{L}) E$ onto $\left(\operatorname{Alg} \mathscr{L}_{1}\right) \varphi(E)$ mapping $\mathscr{L} E$ onto $\mathscr{L}_{1} \varphi(E)$.

(2) $\varphi$, restricted to $(\mathrm{Alg} \mathscr{L})(I-E)$, is an anti-isomorphism of $(\operatorname{Alg} \mathscr{L})(I-E)$ onto $\left(\operatorname{Alg} \mathscr{L}_{1}\right) \varphi(I-E)$ mapping $\mathscr{L}(I-E)$ onto $\mathscr{L}_{1}^{\perp} \varphi(I-E)$.

Proof. Recall (Proposition 2.11) that there are projections $E_{+}, E_{-}$and $P_{0}$, in $\mathscr{L} \cap \mathscr{L}^{\perp}$, such that $I=E_{+}+E_{-}+P_{0}$ and $\varphi\left(\mathscr{L} E_{+}\right)=\mathscr{L}_{1} \varphi\left(E_{+}\right), \varphi\left(\mathscr{L} E_{-}\right)=$ $\mathscr{L}_{1}^{\perp} \varphi\left(E_{-}\right)$and $\mathscr{A} P_{0} \subseteq \mathscr{L}^{\prime} P_{0}$ (where $\mathscr{A}=\operatorname{Alg} \mathscr{L}$ ). Since we can decompose $\mathscr{A}$ as the direct sum of $\mathscr{A} E_{+}, \mathscr{A} E_{-}$and $\mathscr{A} P_{0}$, it suffices to prove the result for the restrictions of $\varphi$ to each of these algebras.

For $\mathscr{A} E_{+}$we can assume $E_{+}=I$ and use Proposition 2.15 (with $\psi$ as in the discussion preceding Lemma 2.12) to conclude that $\psi$ is multiplicative and, thus, so is $\varphi\left(\right.$ as $\varphi(T)=V^{*} \psi(T) V$ and $V$ is unitary).

For $\mathscr{A} E_{-}$we can assume that $E_{-}=I$, i.e. $\varphi(\mathscr{L}) \subseteq \mathscr{L}_{1}^{\perp}$. Fix an involution $J$ of $H$, i.e. an isometric conjugative linear mapping $J$ of $H$ onto $H$ such that $J^{2}=I$. Then it is easy to check that the map $T \mapsto J T^{*} J$ is a *anti-isomorphism of $B(H)$ onto itself. Define $\psi_{0}(T)=\varphi\left(J T^{*} J\right)$ for $T \in$ $(\operatorname{alg}(J \mathscr{L} J))^{*}=\operatorname{Alg}(J \mathscr{L} J)^{\perp}$. This defines a Jordan partial *-isomorphism from $\operatorname{Alg}(J \mathscr{L} J)^{\perp}$ onto $\operatorname{Alg} \mathscr{L}_{1}$ that maps $I$ into $I$ and $(J \mathscr{L} J)^{\perp}$ into $\mathscr{L}_{1}$. Hence, by the part just proved above, $\varphi_{0}$ is an isomorphism and it follows that $\varphi$ is an anti-isomorphism.

For $\mathscr{A} P_{0}=\mathscr{L}^{\prime} P_{0}$ the result follows from [K, Theorem 10].

Corollary 2.16. Every partial triple product isomorphism of CSL algebras is continuous.

Proof. This follows from Theorem 2.15 and Theorem 3.1 of [GM]. (The argument used in the proof of Theorem 2.15 can be used to show that [GM, Theorem 3.1] holds for anti-isomorphisms.)

Corollary 2.17. Let $\mathscr{L}$ and $\mathscr{L}_{1}$ be commutative subspace lattices and suppose that there is an isometry from $\mathrm{Alg} \mathscr{L}$ onto $\mathrm{Alg} \mathscr{L}_{1}$. If $\mathscr{L}$ is completely distributive, then so is $\mathscr{L}_{1}$ and the results of [MT2] can be applied.

Proof. This follows from Theorem 2.15 and [GM, Corollary 2.2]. 
Remark. Combining Theorem 2.15 (for an isometry $\theta$ ) with Theorem 2.1) of [DP] provides an alternative proof of the main result of [MT2] (see Theorem 1.2).

\section{REFERENCES}

[AS] J. Arazy and B. Solel, Isometries of non-self-adjoint operator algebras, J. Funct. Anal. 90 (1990), 284-305.

[A] W. Arveson, Operator algebras and invariant subspaces, Ann. of Math. (2) 100 (1974), 433-532.

[D] K. R. Davidson, Nest algebras, Research Notes in Mathematics, Pitman, New York, 1988.

[DP] K. R. Davidson and S. Power, Isometric automorphisms and homology for non-self-adjoint operator algebras, Preprint.

[GM] F. Gilfeather and R. Moore, Isomorphisms of certain CSL algebras, J. Funct. Anal. 67 (1986), 264-291.

[K] R. Kadison, Isometries of operators algebras, Ann. of Math. (2) 53 (1951), 325-338.

[KR] R. Kadison and J. R. Ringrose, Fundamentals of the theory of operator algebras, vol. 1, Academic Press, 1983; vol. 2, Academic Press, 1986.

[LL] C. Laurie and W. E. Longstaff, A note on rank one operators in reflexive algebras, Proc. Amer. Math. Soc. 89 (1983), 293-297.

[MT1] R. L. Moore and T. T. Trent, Isometries of nest algebras, J. Funct. Anal. 86 (1989), 180-210. [MT2] _ Isometries of certain reflexive operator algebras, Preprint.

Department of Mathematics, University of North Carolina at Charlotte, CharLOTTE, North Carolina 28223

Current address: Department of Mathematics, Technion-Israel Institute of Technology, Haifa 32000, Israel

E-mail address: mar3600@technion.bitnet 